\title{
Intertextualité et archi-iconicité : le cas des représentations scientifiques de la réaction antigène-anticorps.
}

Intertextuality and Archi-iconicity: The Case of Scientific Representations of the Antigen-Antibody Reaction

Alberto Cambrosio, Daniel Jacobi et Peter Keating

\section{OpenEdition}

Journals

Édition électronique

URL : http://journals.openedition.org/edc/161

DOI : 10.4000/edc.161

ISSN : 2101-0366

Éditeur

Université Lille-3

Édition imprimée

Date de publication : 1 décembre 2004

ISBN : 2-9514961-5-X

ISSN : 1270-6841

Référence électronique

Alberto Cambrosio, Daniel Jacobi et Peter Keating, «Intertextualité et archi-iconicité : le cas des

représentations scientifiques de la réaction antigène-anticorps. », Études de communication [En ligne], 27 | 2004, mis en ligne le 14 octobre 2008, consulté le 01 mai 2019. URL : http://

journals.openedition.org/edc/161; DOI : 10.4000/edc.161

Ce document a été généré automatiquement le 1 mai 2019.

(c) Tous droits réservés 


\section{Intertextualité et archi-iconicité : le cas des représentations scientifiques de la réaction antigène-anticorps.}

Intertextuality and Archi-iconicity: The Case of Scientific Representations of the Antigen-Antibody Reaction

Alberto Cambrosio, Daniel Jacobi et Peter Keating

\section{Les sciences sociales aux prises avec les textes scientifiques}

L'approche de la communication scientifique par les sciences sociales souffre paradoxalement d'un trop plein et d'un trop peu. Un trop plein, puisque au cours de ces trente dernières années, une cohorte plutôt bigarrée de chercheurs a jeté son dévolu sur le texte scientifique: sociologues, philosophes et historiens des sciences évidemment, pour qui les archives et les textes sont des matériaux d'études ordinaires. Mais d'autres chercheurs n'ont pas hésité à s'intéresser aux propriétés stylistiques et rhétoriques des discours scientifiques ${ }^{1}$. Parmi eux, quelques linguistes ont consacré quelques études spécifiques aux textes scientifiques (Swales, 1990, 1998 ; Hyland, 1998 ; Mortureux, 1997). On trouve enfin des chercheurs en communication qui ont appliqué les méthodes d'études des médias ou de l'opinion aux traitements des controverses engendrées par les applications des sciences: des petites communautés de chercheurs se rencontrent régulièrement autour de thèmes Science, Technologie, Société (STS) pour dresser des sortes de fresques décrivant la nature des représentations sociales lors de cas particuliers d'irruption de la science dans l'actualité : le SIDA, les OGM, etc. ${ }^{2}$. 
2 Un trop peu côtoie pourtant cette masse conséquente de publications consacrées aux documents qualifiés de scientifiques. Notons, par exemple, que ces travaux ne sont pas parvenus à ébranler la considération massivement positive dont le texte scientifique fait l'objet. Par ailleurs, ce type de recherche affronte une sorte de paradoxe: le chercheur qui rédige une étude sur le discours scientifique est un peu dans une posture d'autoanalyse. Il est conscient des liens et des implicatures propres au genre: il sait qu'il produit, à son tour, de la science.

3 Dernière préoccupation, dans le cas des sciences de la vie, la recherche prend pour objet des documents scriptovisuels et pas seulement des textes ${ }^{3}$. Il s'agit de rendre compte des caractéristiques d'un énoncé parsemé de plages visuelles originales: l'imagerie scientifique. L'imbrication du donné à lire et du donné à voir, la disposition des images sur le support, leur renvoi à l'énoncé et, réciproquement, leur mobilisation dans le fil du discours, produisent du sens. Le reconstruire et l'interpréter est l'une des tâches majeures des chercheurs qui étudient ces dispositifs de communication.

\section{Intertextualité et transtextualité}

4 Dans une série de recherches en partie déjà publiées ou, pour d'autres, encore à venir, nous avons entrepris une exploration de l'imagerie immunologique publiée dans des documents scientifiques: comment, depuis 1900, les représentations scientifiques de la réaction antigène-anticorps (elle est historiquement au centre de toute théorie immunologique) ont-elles évolué ? Quels sont les différents modèles visuels qui se sont succédé? Quelles sont les propriétés sémiotiques de ces dispositifs d'argumentation visuelle $^{4}$ ? La lecture et l'interprétation sémiotique, aussi précises et raffinées soient-elles, ne peuvent évidemment être dissociées du regard historique et sociologique qui, tout autant qu'elles, conduit et oriente cette recherche collective. Cette perspective a comme conséquence que la recherche sur la communication scientifique ne porte donc plus sur des énoncés autonomes mais sur un ensemble considérable dans lequel la décision d'isoler des documents suppose une conscience claire des différents niveaux auxquels l'analyse est susceptible de se déployer.

5 Faute de pouvoir décrire et commenter en détail les méthodes de recherche (elles empruntent aussi bien à la sociologie et à l'histoire des sciences qu'à la communication), dans cet article nous ne commenterons spécifiquement que la place de l'approche communicationnelle. À cet effet, un détour théorique s'impose. En suivant Genette (1982), et en adaptant son questionnement à l'univers des textes scientifiques, dès lors qu'il pose la question qu'est-ce qu'un texte, on en vient presque aussitôt à différencier puis à catégoriser différents types d'écrits: articles, revues de la question, communications reproduites dans les actes de congrès, pre-prints en ligne, notes de recherche, chapitres de manuels universitaires... Ces différences, loin d'être marginales, questionnent la notion de situation de communication: en quoi l'écrivant scientifique inclut intuitivement la singularité de cette situation dans la structure ou la rhétorique du document qu'il élabore ? De la même façon, en quoi cette situation influence la nature de l'imagerie qu'il produit, fait produire et mobilise?

6 Toujours en suivant Genette, dans ses travaux sur la poétique et les genres littéraires, convenons de distinguer trois dimensions distinctes du texte ${ }^{5}$. Sans nécessairement reprendre sa terminologie qui, comme on sait, est assez fluctuante car toujours provisoire 
et réinterrogée, est-il possible d'appliquer un tel questionnement à l'univers du document scientifique?

7 Genette distingue: "la relation d'inclusion qui unit chaque texte aux divers types de discours auxquels il ressortit» (Genette, $2004: 80$ ). Ces qualités propres du texte, qu'il nomme son architextualité, signalent d'emblée et sans équivoque son appartenance à un genre dans lequel il apporte une contribution spécifique. Ce qui a comme conséquence qu'un texte scientifique renvoie aux textes scientifiques déjà publiés ou même qu'il tente d'anticiper sur les textes scientifiques encore à venir. Cette architextualité fait qu'un scientifique reconnaît dans n'importe quel discours son appartenance à un genre dès lors qu'il en parcourt les premières lignes. Mais, note l'auteur, cette qualité ne saurait être séparée de ce qu'il nomme la transtextualité. Ce néologisme englobe, chez Genette, ce que d'autres chercheurs appellent l'intertextualité, mais que, pour sa part, il enrichit de deux prolongements : la para- et la métatextualité ${ }^{\text {. }}$

\section{Le cas de l'imagerie}

8 Ce modèle, pensé et formalisé pour la littérature, a-t-il une quelconque validité dès lors qu'on envisage l'univers scriptovisuel des communications scientifiques? Si personne ne peut douter de l'importance des traces intertextuelles dans le discours scientifique (il suffit de consulter la liste fort longue des références bibliographiques et du nombre de chercheurs, voire de citations, mobilisés dans le texte lui-même), que dire de le méta et de la paratextualité ?

9 Le paratexte au sens de Genette (en tout cas celui qu'il spécifie dans Genette, 1979) correspond à tout l'appareil pragmatique et déictique qui met en scène le texte (titre, collection, quatrième de couverture, résumé, biographie de l'auteur, vignette colorée de la une...). De ce point de vue, le texte scientifique est tout autant paratextualisé que l'œuvre littéraire : éditorial, place dans le sommaire, abstract, mention des laboratoires, titres universitaires des chercheurs, nature et réputation d'excellence des organismes qui ont financé les recherches, mention des circonstances (date et lieu) dans lequel le texte a été produit, etc.

Est-il pour autant métatextualisé ? La dimension méta suppose une sorte de duplication, un décalage entre le chercheur-auteur et son double l'écrivant. Au moment où le chercheur met en texte le document scientifique qu'il soumet à publication, il peut écrire sur l'écriture, faire montre de recul critique et laisser deviner qu'il n'est pas dupe de la bienséance épistémologique en vigueur... en respectant cependant les normes. Le double du chercheur qui écrit marque une distance, une sorte d'évaluation vis-à-vis des idées qu'il propose et de la forme qu'il emploie. Cette posture métatextuelle n'est pas habituelle. On la repère, en tout cas pour les textes rédigés en langue française, par l'occurrence d'embrayeurs comme nous, on ou je dans un discours dans lequel ces pronoms ne renvoient pas à l'auteur lui-même ou au petit groupe des signataires.

Il faudrait introduire pourtant un distinguo entre métatextualité interne et métatextualité seconde. Un discours scientifique évalue les travaux scientifiques qui l'ont précédé, les critique et les commente et, en fin de parcours, avec une modestie plus ou moins feinte, tente de situer sa propre insertion dans le procès continu de production de connaissance. Cette métatextualité structurelle ne préjuge cependant pas de la seconde: c'est-à-dire la place que le modèle proposé parviendra à occuper dans le champ scientifique et surtout l'influence qu'il exercera par ses reprises dans d'autres catégories 
de discours scientifiques: textes de manuels, conférences ou vulgarisation dans les médias qui sont autant de reprises, de paraphrases, de commentaires ou de traductions $\mathrm{du}$ discours scientifique source. Toutes ces reformulations sont autant de témoins objectifs des procédures d'appropriation par différentes catégories d'utilisateurs des textes scientifiques ${ }^{7}$. Bref, dès lors qu'on explore l'univers des discours scientifiques avec cette perspective interdiscursive et dialogique, il s'ouvre sans résistance outrancière aux concepts propres à la sémiotique de la littérature.

Reste pourtant une dimension que la dictature de l'écrit tend en permanence à faire oublier : la mobilisation des plages visuelles dans les documents scientifiques. Notre pari est donc double : non seulement nous empruntons une théorie propre à la littérature (et pas aux sciences), mais de plus il s'agit de l'imagerie et pas du seul texte nu. Faute de place, nous ne discuterons que d'une seule idée (même s'il est difficile de la dissocier totalement de la transtextualité de Genette) que nous nommons l'archi-iconicité.

\section{Les représentations de la réaction antigène-anticorps comme tribut de l'archi-iconicité}

13 Paul Erhlich (1854-1915) donne, en 1900, une conférence à la Société royale de Londres (Ehrlich, 1900). Cette conférence est célèbre car la théorie qu'il y expose est considérée comme une sorte de préfiguration des théories immunologiques modernes (théorie sélective ou clonale). Cette vision prophétique et qui suscitera de vives discussions dans les années suivantes a été présentée avec une série de dessins. Ces planches sont devenues, à cause de la finesse du dessin comme par leur place singulière dans l'histoire des sciences, une sorte d'image fétiche des théories immunologiques actuelles. D'autres dessins ont été produits en appui des conceptions que Paul Ehrlich a proposées à ses contemporains. Nous allons essayer de montrer de quelles sources elles proviennent et pourquoi elles façonnent un modèle auquel toute illustration dans le domaine de l'immunologie sera, pendant près d'un siècle, plus ou moins redevable.

14 Les dessins que propose Ehrlich possèdent trois propriétés nettement visibles :

1. - ils ont un caractère très marqué d'animalité et leur étrangeté renvoie au monde sousmarin encore mal connu au début du XXe siècle ;

2. - observés en détail, ils font toujours et soigneusement apparaitre un rapport d'emboîtement de type mâle femelle;

3. - certains dessins sont organisés en une seule figure qui correspond à une scansion, une représentation conventionnelle singulière de l'espace-temps.

\section{Aux frontières du vivant : l'animalité sous marine}

Bernardino Fantini dans son survol historique de la création, en 1872, de la Station Zoologique de Naples par le savant allemand Anton Dohrn (1840-1909), et de son développement, fait état de la fascination pour la vie marine qu'ont éprouvée naturalistes et philosophes depuis les temps anciens. La mer est non seulement une déesse mythique, mais aussi la source de la vie. Pour les scientifiques allemands du XIXe siècle, faisant suite au physiologiste et théoricien Johannes Müller (1801-1858), la recherche sur les organismes marins était la clé permettant d'élucider les concepts biologiques fondamentaux. Au cours de cette période marquée par le développement du darwinisme, 
la mer représentait le lieu par excellence pour la recherche des formes élémentaires de vie. Ainsi, elle était considérée comme une source privilégiée de connaissances, modèles et objets expérimentaux pouvant servir à élucider les problèmes biologiques fondamentaux, notamment l'organisation des systèmes vivants, l'embryogenèse, la physiologie générale, l'évolution et la phylogénie (Fantini, 2000 : 524).

Cette fascination pour la mer était partagée par les savants d'autres pays, des laboratoires de biologie marine ayant été ouverts au cours de la même période aux Pays-Bas, en France et, par la suite, aux États-Unis, alors que des aquariums accessibles à un large public étaient inaugurés dans différentes villes, notamment à Berlin. Institution indépendante consacrée à la recherche et ouverte aux savants du monde entier, la Station Zoologique de Naples fut équipée des microscopes les plus avancés produits par la technologie allemande. Ses activités ne se limitaient pas à la morphologie des animaux et des plantes marines, mais incluaient aussi la physiologie, l'embryologie, voire même, en 1887, à la suite d'une visite effectuée par Robert Koch (dans le laboratoire duquel Ehrlich avait travaillé), la bactériologie.

FIgURE 1 DEUX DESSINS D'ANTICORPS

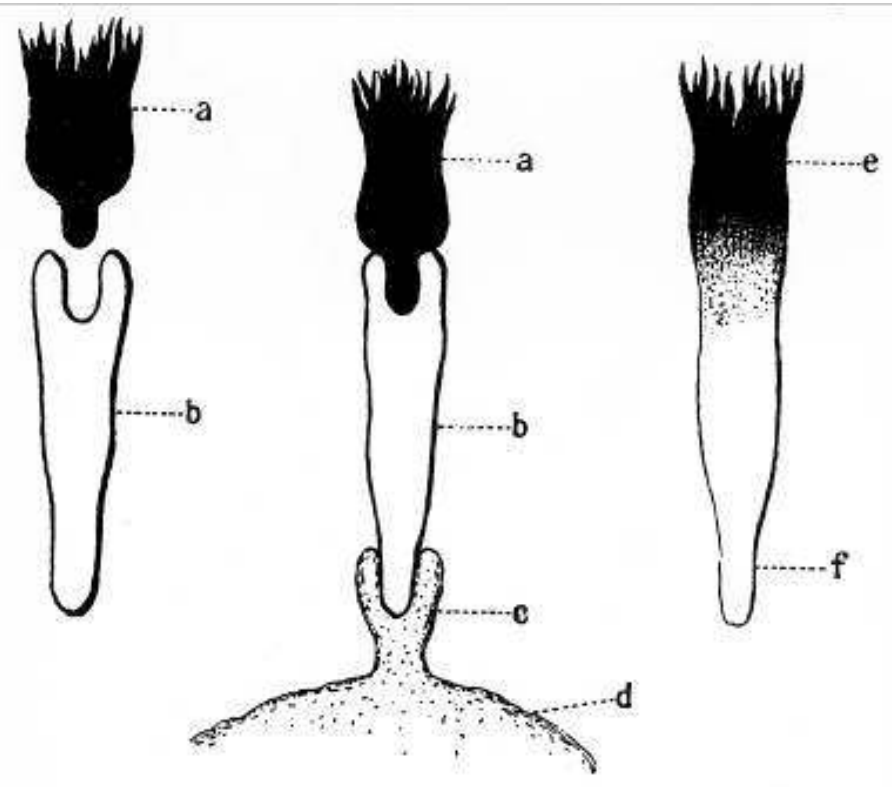

a) Complement; b) Zwischenkörper (Immunkörper); e) Receptor; d) Theil einer Zelle; e) toxophore Gruppe des Toxins; f) haptophore Gruppe. 


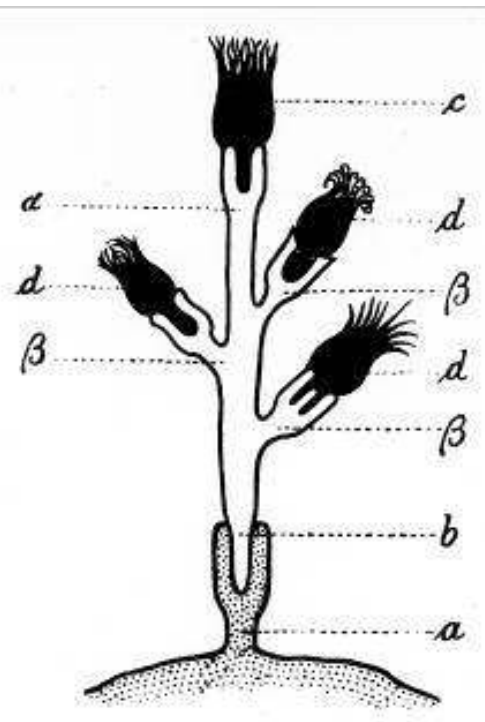

a) Receptor der Zelle. - b) Haptophore Gruppe des Amboceptors. - c) Dominantes Complement. - d) Nichtdominante Complemente.

Comptementophile Gruppen des Amboeeptors: a) für das dominante Complement, $-\beta$ ) für die nichtdominanten Complemente.

Lorsque Ehrlich esquisse (et fait redessiner par un professionnel placé sous étroite surveillance) les formes des corpuscules (les anticorps) qu'il ne peut pas voir (sauf indirectement par les effets d'agglutination qu'ils provoquent) et dont la nature biochimique, voire même l'existence, était à cette époque loin d'être établie, ce sont tout naturellement des formes d'animalcules sous-marins qui viennent sous la mine ${ }^{8}$. Le dessin de gauche de la figure 1 (Ehrlich et Morgenroth, 1900 : 681) fait apparaitre trois entités soigneusement distinguées par la forme du trait et leur valeur distincte, entités que le fléchage anatomique désigne et dénomme. En blanc l'anticorps, en noir la silhouette du complément qui évoque une corolle finement dentelée. Une sorte d'invagination de la cellule (antigène) au milieu et en bas la fait ressembler à un fond rocheux. Le dessin fait sentir qu'il ne s'agit que d'un fragment d'un élément continu plus vaste et un délicat semis lui confère une nuance de gris qui souligne une rupture de milieu entre intérieur et extérieur.

Le dessin central de la figure de gauche et le dessin plus complexe de celle de droite (Ehrlich et Marshall, 1902 : 585) confèrent à ces figures leurs silhouettes qui les ancrent dans le répertoire sous-marin. L'ondoiement souple du trait et les petites corolles de fins tentacules y contribuent fortement. On note sur ces deux dessins le double emboîtement rigoureux mâle femelle de l'anticorps dont la silhouette vide (blanche) souligne la complémentarité parfaite avec le complément (noir) situé au-dessus et la cellule (grisée) en dessous.

C'est la vogue et l'attirance pour le mer qui permettent de mieux saisir la décision d'Ehrlich d'attribuer une animalité sous-marine à ses dessins d'anticorps. La mer est le répertoire iconique spontané qui s'impose au chimiste Paul Ehrlich dès lors qu'il s'agit d'évoquer du vivant. 


\section{L'analogie clef-serrure}

D'après l'Oxford English Dictionary, l'expression combinée "clef et serrure » a été utilisée fréquemment (en anglais) au sens aussi bien littéral que figuré, ce dernier renvoyant d'abord à l'idée de garder quelque chose en sécurité et, plus récemment, aussi à l'idée d'une complémentarité structurelle ou d'une spécificité mutuelle entre deux éléments. Une serrure, par définition, ne peut être pénétrée que par une seule clef; et une clef ne peut commander l'ouverture que d'une seule serrure. Dans ce dernier sens, le premier exemple mentionné par l'OED (en allemand) est justement le fameux passage de l'article de 1894 du futur prix Nobel (1902) de chimie Emil Fischer, où celui-ci affirme que "pour utiliser une image, l'action chimique d'une enzyme sur son substrat exige que ces deux substances soient adaptées l'une à l'autre comme une serrure à sa clef ».

FIgURE 2 LA PRODUCTION D'ANTICORPS SELON EHRLICH EN 1900

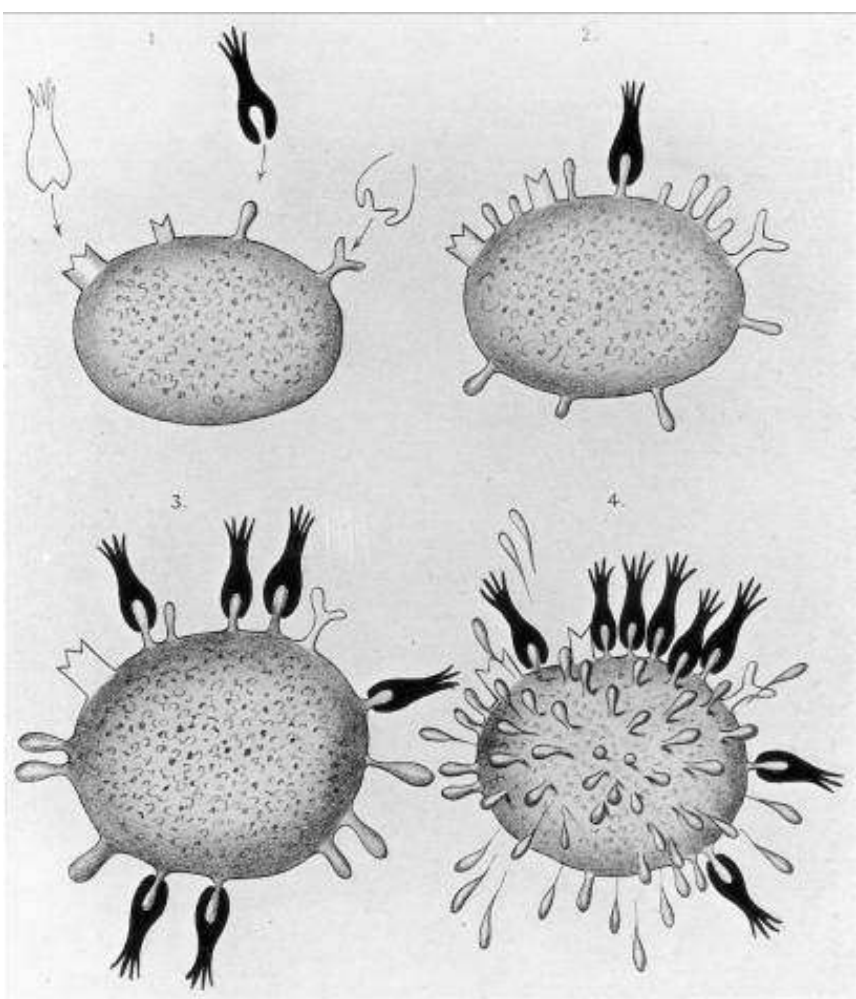

Déjà dans les dessins précédents, d'inspiration sous marine, on a pu remarquer les emboîtements mâle-femelle que les contrastes de blanc et gris soulignent et mettent en valeur. Dans la figure 2, les moyens sémiotiques sont de même nature. Mais le premier dessin de cette planche, celui qui est marqué en haut du chiffre 1, détaille le mode de fixation antigène-anticorps. Les protubérances à la surface de la cellule grise, qui, comme le montre le dessin marqué par le chiffre 4, vont se transformer en anticorps une fois détachés de la cellule, se combinent avec des toxines ou antigènes (en noir), sortes de petites entités dynamiques et qui semblent comme circuler (ou nager?) autour de la cellule de forme sphérique. Ces toxines sont munies, à l'une de leurs deux extrémités, d'une invagination de forme spécifique destinée à leur permettre de s'emboîter exactement sur l'une des protubérances de la grosse sphère grumeleuse grise. Un dispositif déictique (en l'occurrence une petite flèche) attire l'attention et souligne le 
mode d'association : chacune des toxines se fixe électivement sur la seule aspérité avec laquelle elle peut s'ajuster. À cet effet, le dessinateur a ajouté à la toxine noire, la silhouette au trait de deux autres types d'antigènes dont l'un est seulement esquissé, précisément pour mieux faire apparaître la forme spécifique d'une extrémité doublement invaginée et destinée à se fixer à une protubérance en forme de $Y$.

Afin de mieux cerner le rôle et la dynamique de cette analogie mécanique on peut se rapporter aux travaux de l'une des figures majeures de l'analyse des textes scientifiques dans le monde anglo-saxon, Charles Bazerman, et notamment à son étude comparative de trois articles dans trois domaines distincts : les sciences naturelles (le célèbre article de Watson et Crick proposant une structure en double hélice pour l'ADN), les sciences sociales et la critique littéraire. D'après Bazerman (1982: 18-55), le modèle de l'ADN proposé par Watson et Crick en 1953 se fonde sur l'hypothèse, partagée par les chercheurs, d'après laquelle les substances chimiques se caractérisent par une forme géométrique qui leur est propre et qui préexiste à toute interprétation humaine. La structure en double hélice est donc acceptable si elle correspond à la structure "véritable » de la molécule, l'article devant apporter des preuves convaincantes d'une telle correspondance.

Bazerman oppose ensuite cette situation au recours très fréquent à la métaphore (il s'agit en réalité non pas d'une métaphore au sens strict mais d'une analogie) qui caractérise l'argumentation textuelle des sciences sociales et que l'on repère par la présence de formules du type "peut être comprise comme...» ou «se laisse concevoir comme...» ( comme est alors un marqueur de comparaison). Les objets des sciences sociales étant controversés, imprécis, et/ou difficiles à saisir, le chercheur procède en établissant des correspondances avec des objets mieux connus ou mieux définis, sans que cela aboutisse à l'attribution d'une signification précise aux objets qu'il analyse.

Or, si nous examinons l'analogie clef-serrure utilisée par Fischer, nous sommes en présence d'un procédé métaphorique (ou mieux analogique) semblable à celui décrit par Bazerman pour les sciences sociales, mais qui renvoie, dans ce cas, à des structures chimiques comme dans le cas de Watson et Crick. Afin de rendre compte de ce déplacement de rhétorique scientifique, on peut invoquer des facteurs comme la transformation des conditions matérielles et épistémiques de production des faits scientifiques, avec le rôle prépondérant que jouent désormais, après la deuxième guerre mondiale, l'instrumentation et les nouvelles formes d'objectivité qu'elle produit (Baird, 2004), ainsi que l'évolution parallèle et simultanée des stratégies représentationnelles des articles scientifiques (Gross, Harmon et Reidy, 2002). Cet exemple démontre à quel point l'analyse de discours ne saurait être interprétée en dehors de l'intertexte et de son contexte socio-historique de production. Au moment où Ehrlich présente sa conférence, il recourt à un modèle scientifique analogique puisque son domaine de recherche ne dispose pas encore d'un modèle formel abstrait.

En 1894, année où elle fut probablement employée par la première fois par Fischer, à qui Ehrlich l'emprunta quelques années plus tard, l'analogie clef-serrure était un tour de force. D'abord à cause du passage du domaine macroscopique de l'expérience quotidienne au domaine invisible des réactions chimiques. Mais surtout à cause du fait que l'idée qu'il existe des macromolécules caractérisées par une structure à trois dimensions - par une géométrie ou une architecture moléculaire leur étant propre et pouvant donc donner lieu à des arrangements complémentaires dans l'espace - ne faisait pas encore partie de la matrice disciplinaire de ce qu'allait devenir plus tard la biochimie. Ce n'est qu'au cours 
des années 1930 qu'une telle notion commença à être acceptée, d'abord avec difficulté (Cramer, 1997 ; Laszlo, 1986). Cela ne signifie pas que l'idée d'une « forme » des molécules était entièrement nouvelle. Comme le rappelle Laszlo, déjà en 1814 André-Marie Ampère avait évoqué les «dispositions respectives des molécules dont leurs parties intégrantes sont composées» (Laszlo, 2000), et en 1874 van't Hoff avait publié un article sur la structure tétraédrique de l'atome de carbone, allant jusqu'à produire des modèles moléculaires de ces structures. Mais l'utilisation de l'analogie de Fischer, même si on devine sur quels éléments plus ou moins connus cette intuition prend appui, n'en demeure pas moins une extrapolation nouvelle et audacieuse. L'analogie clef-serrure va devenir dès lors un véritable modèle biochimique d'une grande fécondité. Souvent raffiné et complexifié, il ne sera pas démenti.

Ehrlich s'appuie donc sur les arguments de Fischer, ainsi que l'approche qu'ils impliquent, à savoir que les processus biologiques relèvent d'une interprétation chimique. Mais on remarquera que des deux éléments de l'analogie de Fischer, c'est l'aspect adaptation mutuelle de formes géométriques qui prime chez Ehrlich et qui est mis en avant dans sa représentation visuelle de la production des anticorps.

\section{La représentation figurée conventionnelle de l'espace-temps}

On a noté, dans la figure 2, que la fixation de l'anticorps avec l'analogie clef-serrure qu'elle illustre remarquablement est représentée de façon dynamique. En fait, la planche comporte quatre dessins disposés sur deux lignes et que leur numérotation invite à consulter de gauche à droite. On comprend dès lors qu'il s'agit d'une séquence, à savoir quatre étapes successives du mécanisme supposé de la réaction antigène-anticorps. La cellule, dont les protubérances sont bloquées par un nombre croissant de toxines, réagit en multipliant celles-là et en les sécrétant dans le sang où, transformées en anticorps, elles neutralisent les toxines.

Cette représentation imagée de la réaction biologique antigène-anticorps renvoie à une question, plus générale : comment traduire graphiquement les différentes étapes d'un processus biologique qui se déroule simultanément dans l'espace et dans le temps? Pour le lecteur-regardeur d'aujourd'hui, rien de plus simple que de décoder une séquence linéaire d'images juxtaposant, par exemple, les différentes étapes d'une division cellulaire, comme correspondant à une représentation graphique conventionnelle d'une séquence temporelle. Mais, comme le rappelle Marc Ratcliff, une telle convention graphique permettant de lire le temps comme s'il s'agissait d'un texte - tout comme, par ailleurs, d'autres conventions graphiques consistant, par exemple, à entourer d'un cercle toute observation microscopique « instantanée » qui dans le même mouvement marque le changement d'échelle et l'exclusion de la séquence temporelle - est, justement, une convention qu'il s'agit, dès lors, d'historiciser.

Cette inscription dans la diachronie permet d'éviter le piège consistant à attribuer son pouvoir de conviction à des mécanismes cognitifs intemporels. Chacune de ces opérations résulte d'une longue maturation obéissant à un ensemble de contraintes techniques. Le patient travail de construction qui aboutit à une iconographie stabilisée résulte d'un double système de contraintes, à savoir aussi bien des instruments autorisant à produire les images que des techniques typographiques permettant de les reproduire (Ratcliff, 1999). Ainsi, selon Ratcliff, c'est au cours de la deuxième moitié du XVIIIe siècle que les 
naturalistes ont développé la séquence linéaire comme solution graphique au problème de représenter le temps, solution qui ne s'est pas imposée d'emblée.

Sans entrer dans tous les détails d'une démonstration qui est fort riche, limitons nous à rappeler qu'une étape préalable a consisté à concevoir les processus biologiques comme des phénomènes comportant une dimension temporelle, et non pas uniquement spatiale ou morphologique, comme c'était le cas, par exemple, dans la tradition linnéenne du dessin d'histoire naturelle. La mise au point d'une forme standard (dite en cycle) ne s'est pas faite sans de multiples tâtonnements. Par exemple, l'idée de séquence peut être figurée aussi bien du haut vers le bas ou de la droite vers la gauche que de la gauche vers la droite (comme l'écriture, ce qui aujourd'hui nous paraît plus « naturel »). Ou encore, comment faire sentir la différence, au sein d'une même planche, sans délimitation claire, de dessins relevant d'un déroulement séquentiel et d'images statiques?

Un autre indice de cette autonomisation progressive est, dans une première période, le recours systématique aux légendes qui, par la suite, deviendront inutiles pour expliquer comment interpréter les séquences. Bref, les chercheurs sont finalement parvenus à imposer et à adopter la solution de la séquence linéaire qui est encore utilisée de nos jours. Remarquons que si elle renvoie désormais à la temporalité, elle le fait en attribuant à celle-ci une dimension abstraite : elle ne nous donne, en effet, aucune idée de la durée réelle d'un phénomène, mais seulement des étapes à travers lesquelles celui-ci doit passer. Par contre, cette figure permet d'embrasser d'un seul coup d'œil, synoptiquement, le phénomène et en favorise ainsi la mémorisation. C'est sans doute ce qui explique pourquoi cette façon de représenter le temps a par la suite été adoptée par de nombreux manuels de biologie du début du XIXe siècle.

Lorsque Ehrlich a voulu représenter le mécanisme hypothétique de formation des anticorps, il a eu recours à cette tradition d'iconographie séquentielle. Mais il faut faire ici attention : comme le note encore Ratcliff, à l'aide d'exemples historiques, lorsqu'il s'est agi d'appliquer ce type de solution iconographique à de nouveaux objets (et, dans le cas des anticorps à l'époque d'Ehrlich, il s'agit d'objets encore hautement hypothétiques) il a fallu la « reconstruire » afin qu'elle garde sa signification et sa transparence par rapport à ces objets inédits.

Figure vedette ou canevas archi-iconique?

33 Dans l'usage qu'ils font de l'architexte emprunté à Genette, Yves Jeanneret et Emmanuel Souchier redéfinissent plus fermement cette notion en faisant remarquer que le préfixe arché- signifie à la fois origine et commandement :

Tout texte naît d'un architexte qui en balise la structure. Non content de représenter la structure du texte, il en commande l'exécution et la réalisation (Jeanneret et Souchier, 1999 : 103).

La notion d'archi-icône s'inscrit selon nous dans la même perspective. Si les dessins d'Ehrlich ont provoqué, dans un premier temps, de vives polémiques avant d'être repris et utilisés pendant une trentaine d'années, puis plus tard d'être redécouverts, c'est bien parce qu'ils constituent dès leur parution une très robuste figure de référence.

On peut remarquer par ailleurs que dans les reprises et les réutilisations de ces figures par les élèves d'Ehrlich et en particulier dans les manuels, la référence au monde sousmarin a été estompée. On note, sur la figure 3 (gauche : Armand-Delille, 1911 : 33 ; droite : Nedrigailov et Budkewicz, $1912: 697)$, d'une part, une tendance à la schématisation qui, en éliminant les formes animales (21), fait ressortir davantage la complémentarité clef- 
serrure (22). D'autre part, sur la figure de droite, transparaît une seconde tendance : la géométrisation qui rend la représentation définitivement abstraite. La rotation de $90^{\circ}$ que les auteurs ont fait subir aux deux dessins (le mécanisme est représenté horizontalement avec une légende intégrée au dessin) contribue également à cet arrachement d'autant que le contexte est éliminé.

FigURe 3 Le DeVENIR DES dessins PDE PAUL EHRLICH

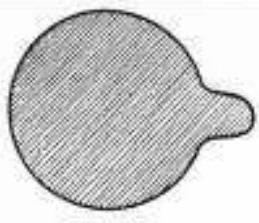

Antigène.

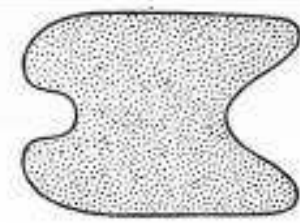

Sensibilisatrice.

= Ambocepteur.

= Fixateur,

= Anticorps.

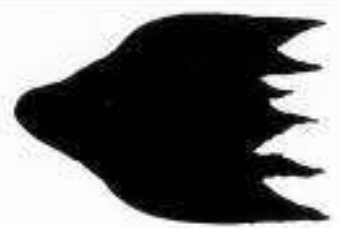

Alexine

$=$ Conplément.

$=$ Cytase.

Fic. 5, - Arant la fixation.

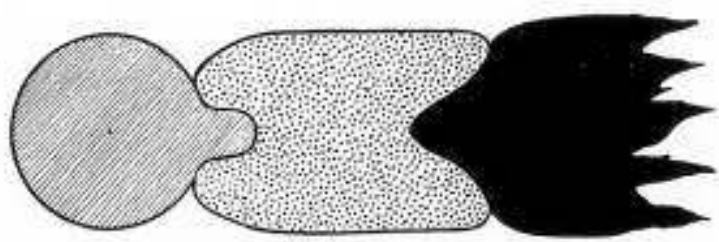

Fu: 6. - Apris la fixation.

\section{Frühere Formel:}

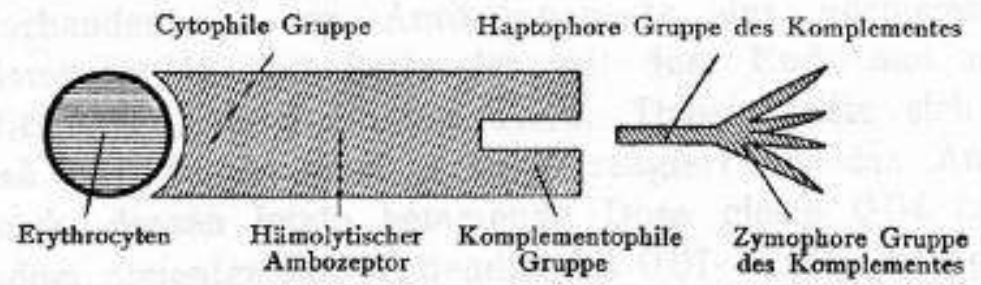

Fig. 1.

\section{Nun kann folgende Formel aufgestellt werden:}

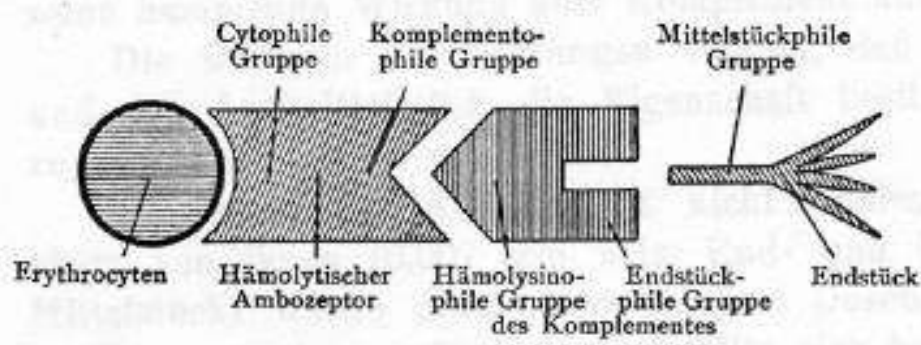

Fig. 2 .

Ainsi, l'étude sémiotique des dessins de Paul Ehrlich, l'interprétation des motifs comme de leurs sources inter-iconiques, le dialogisme transparent avec les chercheurs de son temps, en particulier les chimistes, permettent de retracer leur genèse. Non seulement, il 
propose une interprétation acceptable d'un ensemble de phénomènes macroscopiques de laboratoire comme l'agglutination, mais il les figurabilise de façon convaincante et dynamique. Les conséquences de la diffusion et des multiples débats autour de ces planches sont implicitement d'imposer un modèle crédible de représentation visuelle, une figure obligée de la séquence des transformations aboutissant à la fabrication rapide d'anticorps circulant.

Il s'agit bien de dessins crédibles (et non pas vrais). La distinction entre crédibilité et vérité est aux sources de la sociologie des sciences, dès lors qu'en rejetant tout amalgame facile entre ces deux notions, elle se donne comme objet d'analyser les processus grâce auxquels un argument ou une affirmation donnés deviennent crédibles. La crédibilité d'une figure est indépendante du statut de vérité qu'on lui attribuera par la suite et qui est le résultat, plutôt que la cause, de son gain de crédibilité (Shapin, 1995). L'argumentation visuelle, par son inter-iconicité dialogique, contribue à la production et au maintien de la crédibilité. En particulier, dans le cas d'Ehrlich, l'archi-iconicité et la soumission à un genre (d'autant plus fort qu'il demeure implicite) sont perceptibles. Il élabore une représentation cyclique, qui rend compte du mécanisme de production des anticorps. En même temps, en incluant une schématisation chimique clef-serrure, il révèle sa spécificité. Le processus de crédibilisation tient à la pertinence de cette double option. C'est ce qui explique, non seulement pourquoi Ehrlich est parvenu à convaincre à l'aide de ses planches, mais aussi comment elles sont devenues de facto l'archi-icône que toutes les représentations de la réaction antigène-anticorps seront contraintes de respecter ou de discuter 9 .

De ce point de vue, on peut donc affirmer que la dimension archi-iconique, grâce à laquelle Ehrlich, tout comme ses collègues microscopistes du XIXe siècle explorant la physiologie des cellules, n'a évidemment pas été développée ex-nihilo. Les moyens iconographiques et les modèles visuels disponibles qu'il utilise pour représenter certains éléments temporels se conjuguent avec ce qu'Umberto Eco appelle l'invention d'un code. À savoir la co-production d'un nouvel objet scientifique et des moyens iconographiques pour le représenter, production simultanée du référé et de son référent (Есo, 1979 : 250).

Nous sommes ici confrontés à ce qu'un des pionniers de la sociologie des sciences, Ludwik Fleck, a appelé au cours des années 1930, des «idéogrammes", à savoir des "représentations graphiques de certaines idées et significations, où la signification est représentée comme une propriété de l'objet illustré » (Fleck, 1979 : 173). Mais on peut, chose qui aurait sans doute plu à Fleck, démontrer que ces idéogrammes relèvent d'une archi-iconicité, dont on peut retracer l'histoire pas à pas, tout en apercevant pourquoi ils vont commander les formes des futurs modèles destinés à leur succéder.

\section{BIBLIOGRAPHIE}

Armand-Delille, P.-F., (1911), Techniques du diagnostic par la méthode de déviation du complément, Paris, Masson. 
Baird, D., (2004), Thing Knowledge : A Philosophy of Scientific Instruments, Berkeley, California, University of California Press.

Barthes, R., (1973), Théorie du texte, in : Encyclopedia Universalis, Paris.

Bastide, F. \& Latour, B., (1983), Essai de science fabrication ; mise en évidence du processus de construction de la réalité par l'application de méthodes socio-sémiotiques aux textes scientifiques, Études Françaises 19(2) : 111-33.

Bazerman, C., (1988), Shaping Written Knowledge, Madison, University of Wisconsin Press.

Cambrosio, A., (2000), Argumentation, représentation, intervention : les rôles de l'imagerie dans les discours scientifiques, ASp 27/30 : 95-112.

Cambrosio, A. \& Keating, P., (2000), Of Lymphocytes and Pixels : The Techno-Visual Production of Cell Populations, Studies in History and Philosophy of Biological and Biomedical Sciences $31: 233-70$.

Cambrosio, A., Jacobi, D. \& Keating, P., (2005), Arguing with Images : Pauling's Theory of Antibody Formation, Representations (sous presse).

Cambrosio, A., Jacobi, D. \& Keating, P., (1993), Ehrlich's « Beautiful Pictures » and the Controversial Beginnings of Immunological Imagery, Isis 84 : 662-99.

Cambrosio, A., Jacobi, D. \& Keating, P., (1992), Image et controverse scientifique dans les premières théories immunologiques, in : S. Michaud, J.-Y. Mollier et N. Savy (éds.), Usages de l'image au XIXe siècle, Paris, Créaphis ; 166-81.

Cramer, F., (1997), Emil Fischers Schlüssel-Schloss-Hypothese der Enzymwirkung. 100 Jahre danach, in : H.-J. Rheinberger, M. Hagner et B. Wahrig-Schmidt (éds.), Räume des Wissens, Repräsentation, Codierung, Spur, Berlin, Akademie Verlag ; 191-212.

Eco, U., (1979), A Theory of Semiotics, Bloomington, Indiana University Press.

Ehrlich, P., (1900), On Immunity with Special Reference to Cell Life, Proceedings of the Royal Society of London $66: 424-48$.

Ehrlich, P. \& Marshall, H. T., (1902), Ueber die complementophiles Gruppen der Amboceptoren, Berliner Klinische Wochenschrift 39 : 585-7.

Ehrlich, P. \& Morgenroth, J., (1900), Ueber Hämolysine. Vierte Mitteilung, Berliner Klinische Wochenschrift $37: 681-7$.

Fantini, B., (2000), The « Stazione Zoologica Anton Dohrn » and the History of Embryology, International Journal of Developmental Biology 44 : 523-35.

Fleck, L., (1979/1935), Genesis and Development of a Scientific Fact, Chicago, The University of Chicago Press.

Genette, G., (2004/1979), Fiction et diction. Introduction à l'architexte, Paris, Points.

Genette, G., (1982), Palimpsestes. La littérature au second degré, Paris, Seuil.

Genette, G., (1979), Seuils, Paris, Poétique Seuil.

Gross, A. G., (1996), The Rhetoric of Science. 2nd Edition, Cambridge, MA, Harvard University Press.

Gross, A. G., Harmon, J. E. \& Reidy, M., (2002), Communicating Science : The Scientific Article from the 17th Century to the Present, Oxford, Oxford University Press.

Hyland, K., (1998), Hedging in Scientific Research Articles, Amsterdam, Benjamins. 
Jacobi, D., (2001), Contemporary and Prototypic Figures of Immunology in the Medical Press, in : A. M. Moulin et A. Cambrosio (éds.), Singular Selves. Historical Issues and Contemporary Debates in Immunology, Paris, Elsevier ; 215-27.

Jacobi, D., (1999), La communication scientifique ; discours, figures, modèles, Grenoble, PUG.

Jeanneret, Y., (1994), Écrire la science. Formes et enjeux de la vulgarisation, Paris, P.U.F.

Jeanneret, Y. \& Souchier, E., (1999), Pour une poétique de l'écrit d'écran, Xoana 6 : 97-107.

Kristeva, J., (1969), Séméiotiké. Recherches pour une sémanalyse, Paris, Seuil.

Law, J., (1986), The Heterogeneity of Texts, in : M. Callon, J. Law et A. Rip (éds.), Mapping the Dynamics of Science and Technology, Houndmills, Macmillan ; 67-83.

Laszlo, P., (2000), Playing with Molecular Models, HYLE - International Journal for Philosophy of Chemistry 6(1) : 85-97.

Laszlo, P., (1986), Molecular Correlates of Biological Concepts [Vol. 34A of Albert Neuberger et Laurens L. M. Van Deenen (éds.), Comprehensive Biochemistry], Amsterdam, Elsevier.

Latour, B. \& Bastide, F., (1986), Writing Science. Fact and Fiction : The Analysis of the Process of Reality Construction Through the Application of Socio-Semiotic Methods to Scientific Texts, in : M. Callon, J. Law et A. Rip (éds.), Mapping the Dynamics of Science and Technology, Houndmills, Macmillan ; 51-66.

Mortureux, M.-F., (1997), La lexicologie entre langue et discours, Paris, Sedes.

Moirand, S., (2001), Du traitement différent de l'intertexte selon les genres convoqués dans les événements scientifiques à caractère politique, Semen 13 : 97-117.

Myers, G., (1990), Writing Biology. Texts in the Social Construction of Scientific Knowledge, Madison, University of Wisconsin Press.

Nedrigailov, V. \& Budkewicz, E. V., (1912), Ueber das Antiend- und das Antimittelstück, Zeitschrift für Immunitätsforschung und experimentelle Therapie. I. Teil : Originale 12 : 695-700.

Peytard, J., Jacobi, D. et al., (1984), Français technique et scientifique. Reformulation, enseignement, Langue française 64.

Ratcliff, M. J., (1999), Temporality, Sequential Iconography and Linearity in Figures : The Impact of the Discovery of Division in Infusoria, History and Philosophy of the Life Sciences 21 : 255-92.

Shapin, S., (1995), Cordelias Love : Credibility and the Social Studies of Science Perspectives on Science $3: 255-75$.

Swales, J. M., (1998), Other Floors, Other Voices. A Textography of a Small University Building, Mahwah, NJ, Lawrence Erlbaum, 1998.

Swales, J. M., (1990), Genre Analysis. English in Academic and Research Settings, Cambridge, UK, Cambridge University Press.

\section{NOTES}

1. Cette littérature est surtout en langue anglaise. Voir, par exemple, Bazerman (1988), Gross (1996), Law (1986) et Myers (1990). En langue française, cette literature est plus rare. Une exception, déjà ancienne et qui n'a eu que peu d'héritiers, mérite évidemment d'être saluée. Il s'agit du texte introuvable et mal publié qui a marqué la collaboration entre Latour et la 
scientifique et sémioticienne - trop tôt disparue - Françoise Bastide (Bastide et Latour, 1983). Un version plus complète de cet article est disponible en anglais (Latour et Bastide, 1986).

2. Voir la revue Public Understanding of Science actuellement publiée par Sage à Londres.

3. Sur la notion de scriptovisuel et ses développements comme direction de recherche, voir Jacobi (1999).

4. Notre groupe a déjà publié sur l'imagerie immunologique les articles suivants : Cambrosio, Jacobi et Keating $(2005,1993,1992)$, Jacobi (2001), Cambrosio (2000), Cambrosio et Keating (2000).

5. La notion d'architexte - ou celle non moins cruciale dans ses recherches de paratexte - est fréquemment mobilisée dans les ouvrages peu structurés mais d'une culture éblouissante que Genette publie à intervalles réguliers (on songe ici à ses Figures dont existent déjà plusieurs tomes épais). Voir Genette (2004; 1982).

6. Ce sont Julia Kristeva (1969) et un peu plus tard Roland Barthes (1973) qui les premiers, mais sans formalisation et sans que la méthode fasse école, ont préconisé et défendu l'importance d'une approche intertextuelle.

7. À propos de la reformulation et de ses applications aux discours scientifiques, on se reportera au numéro de la revue Langue Française que l'un de nous a édité avec Jean Peytard (Peytard, Jacobi et al., 1984)

8. Signalons que le plus important concurrent d'Ehrlich en matière d'immunologie, le chercheur franco-russe Élie Metchnikoff (prix Nobel en 1908, partagé avec Ehrlich) effectua ses découvertes concernant la phagocytose comme mécanisme de défense immunologique grâce à des études sur un organisme marin : l'étoile de mer.

9. Faute de place, nous ne pouvons évoquer ici deux prolongements de la recherche. En premier lieu, quelle est la descendance inter-iconique d'un modèle visuel? Quelles sont les transformations faites par Ehrlich et ses élèves aux planches initiales, pour répondre aux critiques de leurs adversaires, pour les reproduire et les diffuser? En second lieu, quelle sera la ré-interprétation méta-iconique de ce nouveau modèle lors de ses reprises entre autres dans la vulgarisation ou l'éducation?

\section{RÉSUMÉS}

Cet article propose d'appliquer à l'imagerie scientifique une approche communicationnelle et sémiotique (inspirée de la théorie du texte de Genette). Une représentation iconique de la réaction antigène anticorps très connue (celle d'Ehrlich proposée en 1900) est étudiée du point de vue de son archi-iconicité. Nous analysons comment tour à tour la référence au monde sousmarin, l'analogie clef-serrure et la représentation conventionnelle du temps contribuent à faire de cette image scientifique un modèle qui aujourd'hui encore inspire les représentations iconiques de cette réaction immunologique essentielle.

The article investigates scientific imagery by resorting to a semiotic and communication studies approach inspired by Genette's theory of texts. In particular, it examines the archi-iconic components of the iconic representation of antigen-antibody reactions first introduced by the German immunologist Paul Ehrlich in 1900. It does so, by showing how the visual reference to the submarine world and organisms, the lock-and-key analogy and graphical conventions for representing time, have made this scientific image into a model that continues to inspire iconic representations of this quintessential immunological reaction. 
INDEX

Mots-clés : imagerie scientifique, médecine, communication scientifique

Keywords : scientific imagery, medecine, scientific communication

\section{AUTEURS}

\section{ALBERTO CAMBROSIO}

Professeur - Social studies of medicine Mcgill University - Montréal - Canada

DANIEL JACOBI

Professeur - laboratoire culture \& communication Université d'Avignon - France

PETER KEATING

Professeur - Département d'histoire UQAM - Montréal - Canada 